Bundesgesundheitsbl 2019 $62: 378$ https://doi.org/10.1007/s00103-019-02897-y Online publiziert: 14. Februar 2019 (c) Springer-Verlag GmbH Deutschland, ein Teil von Springer Nature 2019

CrossMark

Stephan Ehl ${ }^{1}$ Christian Bogdan ${ }^{2,3} \cdot$ Tim Niehues $^{4}$. Gerd Burchard ${ }^{3,5}$. Ulrich Baumann ${ }^{6}$. Jane Hecht ${ }^{7}$. Judith Koch ${ }^{7}$. Johannes G. Liese ${ }^{8}$. Jennifer Neubert ${ }^{9} \cdot$ Miriam Wiese-Posselt $^{7} \cdot$ Fred Zepp $^{3,10}$

${ }^{1}$ Centrum für Chronische Immundefizienz (CCl), Universitätsklinikum Freiburg, Freiburg, Deutschland ${ }^{2}$ Mikrobiologisches Institut - KlinischeMikrobiologie, Immunologie und Hygiene, Universitätsklinikum Erlangen und Friedrich-Alexander-Universität (FAU) Erlangen-Nürnberg, Erlangen, Deutschland

${ }^{3}$ Ständige Impfkommission am Robert Koch-Institut Berlin, Berlin, Deutschland

${ }^{4}$ Zentrum für Kinder- und Jugendmedizin, Helios Klinikum Krefeld, Krefeld, Deutschland

${ }^{5}$ Bernhard-Nocht-Institut für Tropenmedizin, Hamburg, Deutschland

${ }^{6}$ Pädiatrische Pneumologie, Allergologie und Neonatologie, Medizinische Hochschule Hannover, Hannover, Deutschland

${ }^{7}$ Abteilung für Infektionsepidemiologie, Fachgebiet Impfprävention, Robert Koch-Institut Berlin, Berlin, Deutschland

${ }^{8}$ Pädiatrische Infektiologie, Universitätsklinikum Würzburg, Würzburg, Deutschland

${ }^{9}$ Klinik für Kinder-Onkologie, -Hämatologie und Klinische Immunologie, Universitätsklinikum Düsseldorf, Düsseldorf, Deutschland

${ }^{10}$ Zentrum für Kinder- und Jugendmedizin, Universitätsmedizin Mainz, Mainz, Deutschland

\title{
Erratum zu: Impfen bei Immundefizienz
}

\section{Anwendungshinweise zu den von der Ständigen Impfkommission empfohlenen Impfungen. (II) Impfen bei 1. Primären Immundefekterkrankungen und 2. HIV- Infektion}

\section{Erratum zu:}

\section{Bundesgesundheitsbl 2018}

https://doi.org/10.1007/s00103-018-

2761-8

Die Originalveröffentlichung dieses Artikels enthielt einen Fehler in der Autorenliste, in der der beitragende Autor Herr Professor Johannes G. Liese fehlte. Die Autorenliste wurde nun vervollständigt und aktualisiert.

\section{Korrespondenzadresse}

\section{Dr. Jane Hecht}

Abteilung für Infektionsepidemiologie, Fachgebiet Impfprävention, Robert KochInstitut Berlin

Berlin, Deutschland

HechtJ@rki.de 